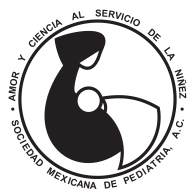

\title{
Prolapso uretral en niñas: reporte de cinco casos
}

\author{
Jenny Elizabeth Arboleda-Bustán, ${ }^{1, *}$ \\ Oriol Martín-Solé, ${ }^{1}$ Sonia Pérez-Bertólez, ${ }^{1}$ Luis García-Aparicio ${ }^{1}$ \\ ${ }^{1}$ Hospital Sant Joan de Déu, Cirugía Pediátrica, Sección de Urología Pediátrica. Barcelona, España.
}

\begin{abstract}
RESUMEN
El prolapso de uretra es una patología poco frecuente. Este trabajo tiene como objetivo describir nuestros casos y compararlo con los estudios publicados. Material y métodos: Estudio descriptivo de las pacientes operadas de prolapso uretral desde 1992 a 2018 de los últimos seis años. Resultados: Se intervinieron cinco pacientes de prolapso uretral. La edad estuvo entre los dos y ocho años. El motivo de consulta fue sangrado. Dos de las cinco presentaron como antecedente reciente infección de vías respiratorias altas. La técnica usada para la corrección es la descrita por Kelly-Burnham; en el seguimiento no hubo complicaciones. Conclusión: EI prolapso de uretra es una patología poco frecuente que se presentó en niñas entre dos y ocho años de edad, el cual requiere un diagnóstico adecuado; el tratamiento más eficaz es quirúrgico.
\end{abstract}

Palabras clave: Prolapso de uretra, niñas, cirugía.

\begin{abstract}
Urethral prolapse is a rare condition. This study aims to describe our cases and compare them with published studies. Materials and methods: Descriptive study, taking as cases all patients operated on for urethral prolapse from 1992 to 2018 in our center in the last six years. Results: Five urethral prolapse patients. Age of our patient was between two and eight years. The reason for consultation was bleeding. Two of the five patients presented a recent history of upper respiratory tract infection. The technique used for the correction was the one described by Kelly-Burnham, in the follow-up patients did not present complications. Conclusion: Urethral prolapse is a rare condition that occurred in girls between 2-8 years of age that requires an adequate diagnosis Surgery is the treatment of choice.
\end{abstract}

Keywords: Urethral prolapse, girls, surgery.

En Pediatría, tiene mayor frecuencia en niñas, con una edad de presentación de tres a nueve años. ${ }^{1} \mathrm{La}$ etiología incluye inadecuada unión muscular perineal, pobre unión entre las capas musculares de la uretra y, aumento de la presión intraabdominal. La teoría congénita atribuye a las estructuras pélvicas débiles, mientras que la teoría adquirida sugiere como posibles causas al trauma, procesos infecciosos del tracto urinario o deficiencia de estrógenos. Además, de cualquier condición que aumente la presión intraabdominal como el parto, violación, constipación, tos o sobrepeso/ obesidad. ${ }^{1-13}$ Otras hipótesis incluyen: desorden neuromuscular, mala posición de la uretra o inadecuada elasticidad de los tejidos. ${ }^{7}$

El diagnóstico diferencial en niñas incluye ureterocele prolapsado, condiloma, quiste periuretral, pólipos, sarcoma botrioide, carúncula uretral, sangrado

\footnotetext{
* Correspondencia: JEAB, elizabethab2011@gmail.com Conflicto de intereses: Los autores declaran que no tienen. Citar como: Arboleda-Bustán JE, Martín-Solé O, Pérez-Bertólez S, García-Aparicio L. Prolapso uretral en niñas: reporte de cinco casos. Rev Mex Pediatr 2019; 86(6):219-222. doi: 10.35366/91872 [Urethral prolapse in girls: report of five cases]
} 
vaginal, traumatismo genital y abuso sexual..$^{3,9,12} \mathrm{El}$ tratamiento médico consiste en baños de asiento y aplicación tópica de crema de estrógenos, esteroides o antibiótico, la eficacia del mismo es de 33 a $38 \%$, según los estudios de Trotman y Holbroo. La cirugía está indicada cuando el tratamiento conservador no es efectivo, hay recurrencia o complicaciones. ${ }^{4}$ Son dos las técnicas quirúrgicas descritas: la resección parcial y la resección completa de la mucosa prolapsada. La cirugía realizada es con la técnica de Kelly-Burnham ${ }^{8}$ (Figura 1), que consiste en colocar una sonda vesical de acuerdo con la edad; luego de referenciar los cuatro puntos cardinales, se suturan los bordes. Las complicaciones incluyen estenosis y disuria.

El presente estudio es la descripción de una serie de cinco niñas con prolapso uretral, quienes han requerido cirugía para su tratamiento; ellas fueron sido atendidas en Hospital Sant Joan de Déu, en Barcelona, entre 1992 y 2018.

\section{RESULTADOS}

Se intervinieron cinco pacientes de prolapso uretral. El motivo de consulta fue tumoración que ocupa la parte central del introito vulvar. En una de ellas se intentó el tratamiento conservador con estrógenos tópicos, pero no dio resultado. Dos presentaron episodios previos de infección respiratoria alta. Todas se resolvieron quirúrgicamente, se mantuvieron hospitalizadas de uno a seis días, la sonda vesical se mantuvo entre dos a seis días, no presentaron estenosis uretral ni otras complicaciones (Tabla 1).

\section{DESCRIPCIÓN DE LOS CASOS}

Caso 1: niña de dos años y 11 meses que acude a urgencias por presentar masa en el introito vaginal. A la exploración física se evidencia prolapso completo de uretra. Se colocó sonda urinaria y se realizó cirugía; permaneció con la sonda y hospitalizada por cuatro días. Durante el seguimiento no ha presentado complicaciones hasta la edad de 16 años.

Caso 2: niña de seis años con antecedentes de una semana previa de bronquitis aguda. Acude a urgencias con un cuadro de sangrado genital. En la exploración física se evidencia prolapso completo de uretra. Se realiza la cirugía. Posterior al procedimiento, no ha presentado complicaciones y al momento tiene 16 años.

Caso 3: niña de ocho años de edad con antecedentes de asma; acude a urgencias por presentar tumoración en introito vaginal. En la exploración física se evidencia prolapso uretral tipo III no reductible. Ingresa y recibe tratamiento quirúrgico. Hospitalizada un día, permanece con sonda urinaria por seis días. En el seguimiento no presenta complicaciones y al momento tiene 12 años.

Caso 4: niña de cinco años de edad sin antecedentes de interés; acude a urgencias con una masa tipo pólipo a nivel del introito vaginal de tres días de evolución. La exploración física fue difícil. Es valorada por Ginecología, quienes realizan vaginoscopía y confirman prolapso uretral. Se decide resolución quirúrgica y
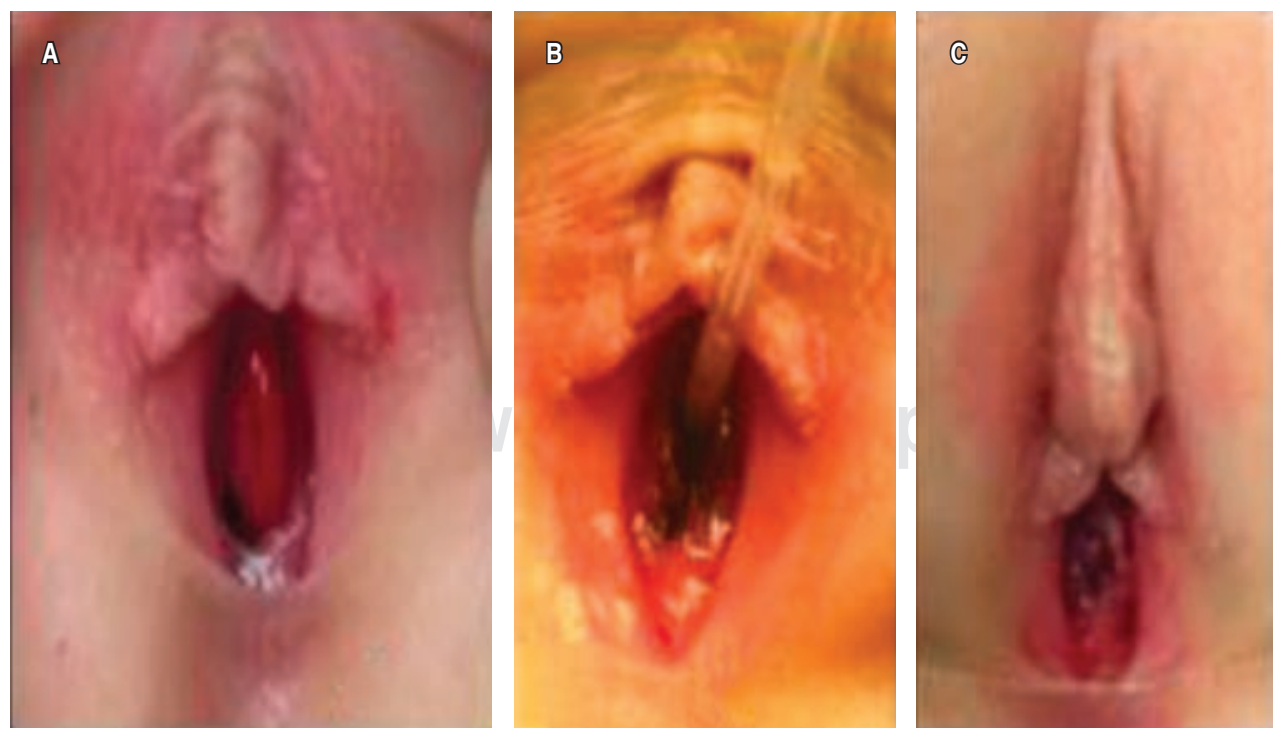

Figura 1:

Prolapso de uretra. A) Examen físico: prolapso uretral. B) Cirugía de Kelly-Burnham. C) Control postquirúrgico al mes. 
Tabla 1: Series de casos descritas sobre pacientes con prolapso uretral.

\begin{tabular}{|c|c|c|c|c|c|c|c|c|c|}
\hline Autores & Año & $\begin{array}{c}\text { No. } \\
\text { pacientes }\end{array}$ & $\begin{array}{l}\text { Edad } \\
\text { (años) }\end{array}$ & $\begin{array}{l}\text { Masa } \\
\mathrm{n}(\%)\end{array}$ & $\begin{array}{c}\text { Sangrado } \\
n(\%)\end{array}$ & $\begin{array}{c}\text { Disuria } \\
\mathrm{n}(\%)\end{array}$ & $\begin{array}{c}\text { Sobrepeso } \\
n(\%)\end{array}$ & $\begin{array}{l}\text { Tratamiento } \\
\text { médico n (\%) }\end{array}$ & $\begin{array}{c}\text { Tratamiento } \\
\text { quirúrgico n (\%) }\end{array}$ \\
\hline Holbrook, et al ${ }^{14}$ & 2012 & 21 & $2-15$ & $8(38.1)$ & $6(28.6)$ & $6(28.6)$ & - & $13(61.9)$ & $8(38.1)$ \\
\hline Ninomiya, et al $\left.\right|^{5}$ & 2017 & 77 & $2-15$ & $25(32.5)$ & $66(85.7)$ & $36(49.7)$ & - & $35(45.4)$ & $42(54.5)$ \\
\hline Wei, et $\mathrm{al}^{13}$ & 2017 & 89 & $6-8$ & 34 (38.2) & $54(60.7)$ & $1(1.1)$ & $56(62.9)$ & $14(15.7)$ & $55(61.8)$ \\
\hline $\begin{array}{l}\text { Arboleda-Bus- } \\
\text { tán, et al }\end{array}$ & 2019 & 5 & $6.2^{*}$ & $4(80.0)$ & $1(20.0)$ & - & $3(60.0)$ & $1(20.0)$ & $5(100.0)$ \\
\hline
\end{tabular}

permanece ingresada con sonda vesical por seis días. En el seguimiento no presenta complicaciones y al momento tiene 10 años.

Caso 5: niña de ocho años de edad que realiza ciclismo e hipismo. Es derivada por presentar sagrado genital de un mes de evolución. Inicialmente fue tratada con estrógenos tópicos, sin ser efectivos después de 15 días. Se decide resolución quirúrgica, se mantiene en hospital durante dos días y con la sonda urinaria una semana. No presenta complicaciones, al momento tiene 10 años.

\section{DISCUSIÓN}

El PU es una entidad descrita principalmente en niñas. Se ha reportado que esta patología se presenta con más frecuencia en edades comprendidas entre los tres y nueve años, lo cual ocurrió también en nuestros casos ya que se presentaron entre los dos y ocho años de edad. El motivo de consulta de las pacientes con PU puede ser: disuria en $47 \%$ de los casos y en $32 \%$ consultan por masas en el introito. ${ }^{6}$ Dos de nuestras pacientes consultaron por masa en el introito y tres por sangrado genital, que nos lleva a realizar el diagnóstico diferencial con traumatismo o abuso sexual. . $^{3,9,14}$

El diagnóstico es clínico, la exploración física es patognomónica con el signo de la dona, confirmándose con la colocación de una sonda vesical. Sin embargo, dado que es una patología infrecuente en la práctica clínica pediátrica, la tasa de diagnóstico correcto en el examen inicial es sólo de $21 \%$, por lo que es común que se confunda con tumores o abuso sexual. ${ }^{5,8,9}$ Además, las pacientes pediátricas a menudo son poco colaboradoras, lo que dificulta el diagnóstico.
El tratamiento conservador es con el uso tópico de estrógenos, corticoides y cremas antibióticas utilizadas como primera línea; empero, la tasa de resolución es de alrededor de $30 \%$ de los casos. Un estudio multicéntrico en Japón refiere que obtienen mejores resultados con el tratamiento quirúrgico. ${ }^{5}$ En nuestro estudio sólo una paciente se trató con estrógenos tópicos, y al no tener efecto se decidió la resolución quirúrgica, lo cual concuerda con lo reportado en la literatura.

Por último, en la Tabla 1 se hace una comparación de las características de la presente serie de cinco casos, con otras ya publicadas de pacientes con PU. ${ }^{5,13,14}$

\section{AGRADECIMIENTO}

Expresamos nuestro agradecimiento al Hospital Sant Joan de Déu por las facilidades prestadas para el presente trabajo.

\section{REFERENCIAS}

1. Lowe FC, Hill GS, Jeffs RD, Brendler CB. Urethral prolapse in children: insights into etiology and management. J Urol. 1986; 135(1): 100-103.

2. Mitre A, Nahas W, Gilbert A, Glina S, Saiovici S, Mazzucchi E et al. Urethral prolapse in girls: familial case. J Urol. 1987; 137(1): 115.

3. Agarwal S, Lall A, Bianchi A, Dickson A. Uro-genital bleeding in pre-menarcheal girls: dilemmas of child abuse. Pediatr Surg Int. 2008; 24(6): 745-746.

4. Ballouhey Q, Galinier P, Gryn A, Grimaudo A, Pienkowski $C$, Fourcade $\mathrm{L}$. Benefits of primary surgical resection for symptomatic urethral prolapse in children. J Pediatr Urol. 2014; 10(1): 94-97.

5. Ninomiya T, Koga H. Clinical characteristics of urethral prolapse in Japanese children. Pediatr Int. 2017; 59(5): 578-582.

6. Fernandes ET, Dekermacher S, Sabadin MA, Vaz F. Urethral prolapse in children. Urology. 1993; 41(3): 240-242. 
7. Kondamudi NP, Gupta A, Watkins A, Bertolotti A. Prepubertal girl with vaginal bleeding. J Emerg Med. 2014; 46(6): 769-771.

8. Chiba M, Toki A, Sugiyama A, Suganuma R, Osawa S, Ishii R et al. Urethral caruncle in a 9-year-old girl: a case report and review of the literature. J Med Case Rep. 2015; 9: 71.

9. Vunda A, Vandertuin L, Gervaix A. Urethral prolapse: an overlooked diagnosis of urogenital bleeding in pre-menarcheal girls. J Pediatr. 2011; 158(4): 682-683.

10. Owens SB, Morse WH. Prolapse of the female urethra in children. J Urol. 1968; 100(2): 171-174.
11. Epsteen A, Strauss B. Prolapse of female urethra with gangrene. Am J Surg. 1937; 35: 563-569.

12. Singh MM, Parsekar SS, Nair SN. An epidemiological overview of child sexual abuse. J Family Med Prim Care. 2014; 3(4): 430-435.

13. Wei $Y$, Wu SD, Lin T, He DW, Li XL, Wei GH. Diagnosis and treatment of urethral prolapse in children: 16 years' experience with 89 Chinese girls. Arab J Urol. 2017; 15(3): 248-253.

14. Holbrook C, Misra D. Surgical management of urethral prolapse in girls: 13 years' experience. BJU Int. 2012; 110(1): 132-134. 